\title{
OPEN Multiplanar reformation improves identification of the anterolateral ligament with MRI of the knee
}

\author{
Andreas Hecker ${ }^{1 凶}$, Rainer J. Egli ${ }^{2}$, Emanuel F. Liechti ${ }^{1}$, Christiane S. Leibold ${ }^{1}$ \& \\ Frank M. Klenke ${ }^{1}$
}

The anterolateral ligament (ALL) is subject of the current debate concerning rotational stability in case of anterior cruciate ligament (ACL) injuries. Today, reliable anatomical and biomechanical evidence for its existence and course is available. Some radiologic studies claim to be able to identify the ALL on standard coronal plane MRI sections. In the experience of the authors, however, ALL identification on standard MRI sequences frequently fails and is prone to errors. The reason for this mainly lies in the fact, that the entire ALL often cannot be identified on a single MRI image. This study aimed to establish an MRI evaluation protocol improving the visualization of the ALL, using multiplanar reformation (MPR) with the goal to be able to evaluate the ALL on one MRI image. A total of $47 \mathrm{knee}$ MRIs performed due to atraumatic knee pain between 2018 and 2019 without any pathology were analyzed. Identification of the ALL was performed twice by an orthopedic surgeon and a radiologist on standard coronal plane and after MPR. For the latter axial and coronal alignment was obtained with the femoral condyles as a reference. Then the coronal plane was adjusted to the course of the ALL with the lateral epicondyle as proximal reference. Visualization of the ALL was rated as "complete" (continuous ligamentous structure with a tibial and femoral insertion visible on one coronal image), "partial" (only parts of the ALL like the tibial insertion were visible) and "not visible". The distances of its tibial insertion to the bony joint line, Gerdy's tubercle and the tip of the fibular head were measured. On standard coronal images the ALL was fully visible in 17/47, partially visible in $27 / 47$, and not visible in $3 / 47$ cases. With MPR the ALL was fully visible in 44/47 and not visible in 3/47 cases. The median distance of its tibial insertion to the bony joint line, Gerdy's tubercle and the tip of the fibular head were 9,21 and $25 \mathrm{~mm}$, respectively. The inter- (ICC: $0.612 ; 0.645 ; 0.757$ ) and intraobserver (ICC: $0.632 ; 0.823 ; 0.857)$ reliability was good to excellent. Complete visualization of the ALL on a single MRI image is critical for its identification and evaluation. Applying multiplanar reformation achieved reliable full-length visualization of the ALL in $94 \%$ of cases. The described MPR technique can be applied easily and fast in clinical routine. It is a reliable tool to improve the assessment of the ALL.

The anterolateral ligament (ALL) is subject of the current debate on rotational stability in association with anterior cruciate ligament (ACL) injuries ${ }^{1}$. Although reliable anatomical and biomechanical evidence for its existence is available today ${ }^{2}$, the impact of the ALL on rotational stability has not been fully understood. Furthermore, the indication to reconstruct the ALL has been discussed controversially ${ }^{3}$.

A biomechanical cadaver study reported abnormal knee kinematics in ALL deficient knees when only the ACL was reconstructed and found normalization of the kinematics if an additional modified deep Lemaire or MacIntosh tenodesis was added ${ }^{4}$. Another study that supports the important role regarding rotatory stability found a significant increase of rotatory instability when the ALL was sectioned additional to an ACL deficiency and therefore, claimed the ALL to be an important secondary stabilizer of the $\mathrm{knee}^{5}$. A recent study with a minimum 2 years follow-up was able to link this biomechanical findings to clinical results. They reported a lower reconstruction failure rate and better clinical outcomes in patients without ALL injury compared to patients with ALL injury on the initial MRI. Anterior stability and pivot-shift interestingly did not differ between those two groups ${ }^{6}$. The surgical decision to additionally perform an anterolateral stabilizing procedure is primarily made based on the clinical finding of rotational instability, i.e. a high-grade pivot shift and the type and level of the sporting activity of the patients ${ }^{7}$. An international consensus paper summarized the possible indications

${ }^{1}$ Department of Orthopaedic Surgery and Traumatology, Inselspital, Bern University Hospital, University of Bern, Bern, Switzerland. ${ }^{2}$ Department of Diagnostic, Interventional and Pediatric Radiology (DIPR), Inselspital, Bern University Hospital, University of Bern, Bern, Switzerland. ${ }^{\boxplus}$ email: andreas.hecker@insel.ch 
for additional anterolateral procedures. They stated that revision ACL reconstruction, high grade pivot-shift, generalized ligamentous laxity, genu recurvatum and young patients returning to pivoting activities may be good candidates for such additional procedures ${ }^{8}$.

The radiological assessment is crucial to distinguish whether rotational instability is due to an ALL injury or another reason such as a meniscal root tear ${ }^{9,10}$. This should ultimately guide the surgical and non-surgical treatment of these patients. For radiologic assessment, an MRI is usually obtained in addition to standard radiographs. Previous studies report ALL identification rates on standard coronal plane MRI images ranging between 11 and $100 \%{ }^{11-13}$. A recent review on the identification of the ALL on MRI highlights this heterogeneity and attributes this to different protocols used for the assessment. Moreover, knee sizes plays an important role regarding length, width and thickness of the ALL ${ }^{14}$. This is further supported by a study of patients younger than 18 years, were the authors stated that identification of the ALL was not possible on MRI in females younger than 7 years and males younger than 6 year. In this study a visualization of the ALL in $70 \%$ was reached after the age of 13 years in both sexes $^{15}$. Most of the studies reporting on the ALL used standard coronal images for the assessment, while some tried to enhance ALL visualization by slightly flexing and externally rotating the knee during image acquisition ${ }^{14}$.

However, identification of the ALL on standard coronal MRI images is challenging and associated with a high level of uncertainty in the discrimination of the ALL and its surrounding structures. This is because the ALL is a fine structure which is usually not aligned precisely to the coronal plane. Furthermore, it merges with the lateral collateral ligament (LCL) near its femoral insertion complicating the discrimination of the two structures proximally. Muramatsu et al. reported 3D-MRI in contrast to the above mentioned 2D analysis allows reliable full length identification of the ALL ${ }^{16}$. To assess the ALL on MRI, a very good anatomical understanding of the anterolateral complex of the knee is required because the capsule, the meniscotibial and meniscofemoral ligaments as well as fibers of the LCL or the iliotibial band may be mistaken as the ALL due to their immediate proximity. Visualization of the whole ALL including its femoral and tibial attachment on one MRI layer seems to be critical for correct identification of the ligament, because if only parts of the ALL are visible, a confusion with other anterolateral structures like the posterior fibers of the iliotibial band, the joint capsule or the meniscotibial and meniscofemoral ligaments is possible. Considering this, a standardized imaging approach using well-defined anatomical landmarks is key but lacking to date. The mentioned anatomic structures are displayed in Fig. 1.

This study aimed to establish an MRI evaluation protocol improving the visualization of the ALL using a standardized multiplanar reformation (MPR) protocol. It was hypothesized that visualization of the ALL can be improved significantly by applying this protocol when compared to the evaluation on standard coronal plane MRI.

\section{Material and methods}

All patients undergoing an MRI performed due to atraumatic knee pain between 2018 and 2019 were retrospectively identified within the picture archiving and communication system (PACS). Only patients analyzed in a $3 \mathrm{~T}$ MRI with a 3-dimensional intermediate weighted (proton weighted) fat-suppressed sequence (3D PD VISTA SPAIR; TR $1300 \mathrm{~ms}$, TE $32 \mathrm{~ms}$, slice thickness $0.7 \mathrm{~mm}$ ) and with a standard coronal reformation (slice thickness $1 \mathrm{~mm}$ ) of this acquisition directly performed at the MRI console were included. MRIs revealing any injury or pathology as well as previous surgery around the knee were subsequently excluded. Due to technical reasons not anonymized MRI data was analyzed first, the anonymization was done when documenting the measurement results. The study was approved by the local ethics committee and handling of the data was performed in accordance with the guidelines of the Declaration of Helsinki and the Swiss human research act. The ethics committee (Cantonal Research Ethics Commission, Bern, Switzerland) waived the need to obtain informed consent in this study, according to Article 34 of the Swiss human research act.

First, the ALL was identified on standard coronal reformations and classified as completely visible, partially visible and not visible (Fig. 2). Complete visibility was defined as identification of the entire ALL including its tibial and femoral insertions on a single MRI layer. Based on the results of anatomical dissections the femoral insertion was identified at the lateral femoral epicondyle and the tibial attachment midway between Gerdy's tubercle and the fibular head ${ }^{17-19}$. Second, the 3D acquisition was analyzed using MPR allowing for free orientation of the axial, coronal, and sagittal image planes.

To create a reproducible starting position, the axial and coronal planes were aligned to the inferior and posterior edges of the femoral condyles, respectively (Fig. 3). Next, the coronal plane was aligned exactly to the course of the ALL. To this end, the center of the MPR coordinate system was shifted to the proximal and posterior edge of the lateral epicondyle (Fig. 4a), because previous studies reported the proximal insertion of the ALL at or in very close proximity to the lateral epicondyle ${ }^{19,20}$. Then the $\mathrm{z}$-axis was tilted in the sagittal image (Fig. $4 \mathrm{~b}$ ), while observing the lateral ligamentous structures in the coronal image until a continuous structure, the ALL, could be identified from the lateral epicondyle to the tibia between Gerdy's tubercle (the attachment site of the iliotibial band) and the tip of the fibular head (the attachment site of the collateral ligament) (Fig. 4c,d). Visualization of the ALL was rated as "complete" (continuous ligamentous structure with a tibial and femoral insertion visible on one reformatted coronal image), "partial" (only parts of the ALL like the tibial insertion were visible) and "not visible". The distances of its tibial insertion to the bony joint line, the midpoint of Gerdy's tubercle and the midpoint of the tip of the fibular head were measured on coronal and axial images.

All measurements were performed twice by an orthopedic surgeon and a radiologist with a minimum time of 2 weeks between the reads.

SPSS statistics was used for data analysis (IBM SPSS Statistics, Version 25 for Windows). Data was not normally distributed and are given as median and range. Intraclass correlation (ICC) was calculated to assess inter- and intra-observer reliability. Two-way mixed measures checked for consistency and ICC is presented with $95 \%$ confidence interval. ICC values of $<0.40$ were rated as poor, between 0.40 and 0.59 fair, between 0.60 


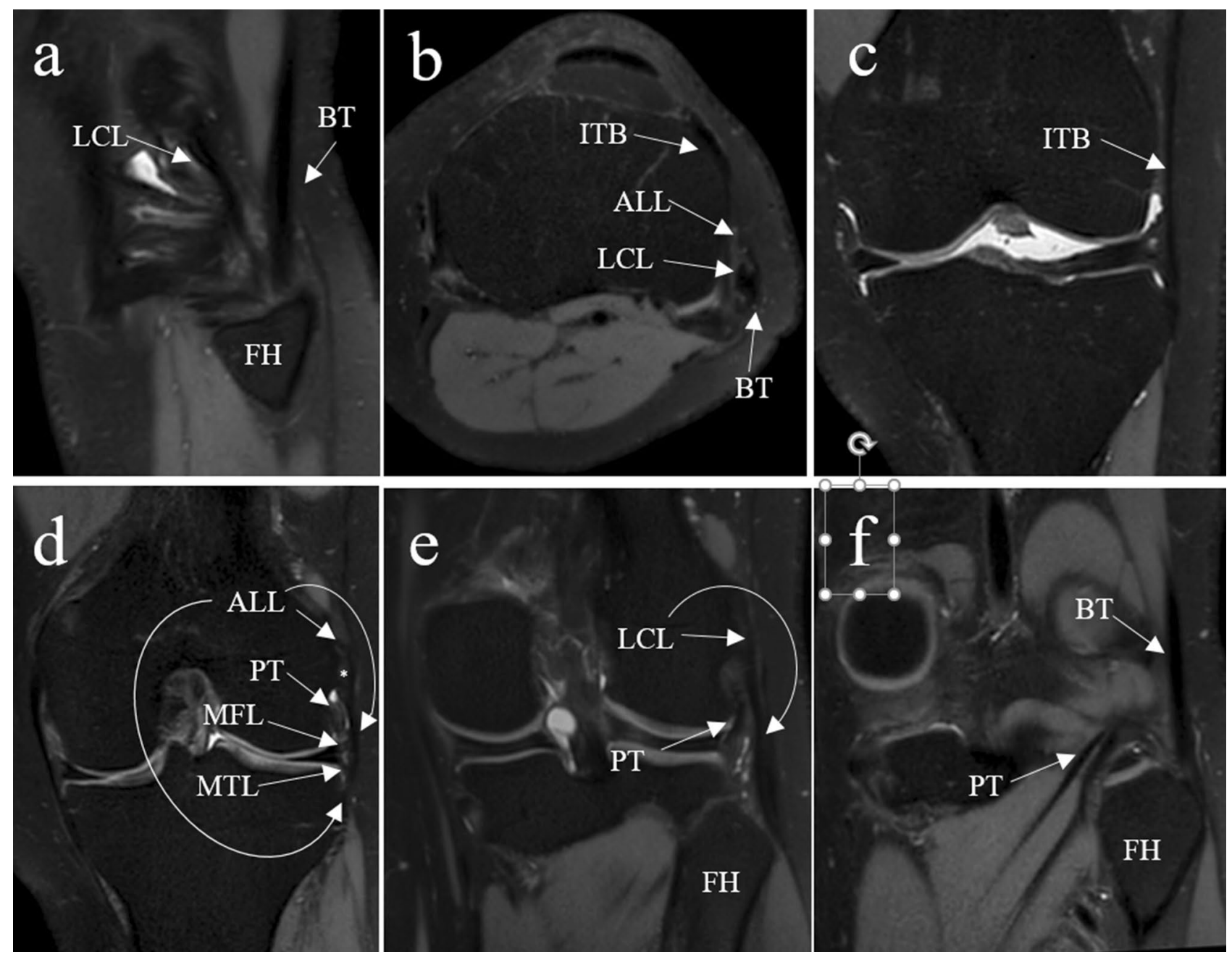

Figure 1. The anterolateral structures are shown in different MRI planes. (a) Represents a sagittal MRI image with the lateral collateral ligament (LCL) and the biceps tendon (BT) inserting at the fibular head (FH). (b) An axial image that displays the insertion of the iliotibial band (ITB) at Gerdy's tubercle as well as the anterolateral ligament (ALL), LCL and BT. (c) Shows the course of the ITB in a coronal image. In image (d) the entire ALL with femoral, tibial and meniscal part is marked as well as the popliteus tendon (PT) and the meniscofemoral (MFL) and meniscotibial ligament (MTL). In this image the lateral epicondyle with the merging fibers of ALL, LCL and MFL is marked with an asterisk. Another coronal image further posterior shows the LCL and the PT (e), while in the last image (f) even further posterior the BT and PT are shown.

and 0.74 good and between 0.75 and 1.00 excellent $^{21}$. McNemar's test was used to compare visibility of the ALL in standard coronal images and after MPR.

Ethical approval. The study was approved by the local ethical review board (Cantonal Research Ethics Commission Bern, Switzerland) Application BASEC-Nr. 2020-01559.

Informed consent. Informed consent was not necessary for this retrospective study according to the swiss human research act.

\section{Results}

47 patients could be included for detailed analysis. 28 patients were women and 19 men, the mean age was 29 (range 20-39) and 24/23 were left/right knees, respectively. On standard coronal images Reader 1 rated the ALL as fully visible in $26 \%(12 / 47)$, partially visible in $66 \%(31 / 47)$, and not visible in $9 \%(4 / 47)$ cases in the first read and as fully visible in $28 \%$ (13/47), partially visible in 64\% (30/47), and not visible in 9\% (4/47) cases in the second read (Table 1). Reader 2 rated the ALL on the same images as fully visible in $40 \%$ (19/47), partially visible in $55 \%(26 / 47)$, and not visible in $4 \%(2 / 47)$ cases in the first read and as fully visible in $49 \%(23 / 47)$, partially visible in $49 \%$ (23/47), and not visible in $2 \%(1 / 47)$ cases in the second read. With MPR Reader 1 rated the ALL as fully visible in $98 \%(46 / 47)$ in both reads and Reader 2 rated the ALL on the same images as fully visible in $87 \%(41 / 47)$ in the first read and in $94 \%(44 / 47)$ in the second read. The rate of full visibility of the ALL improved 


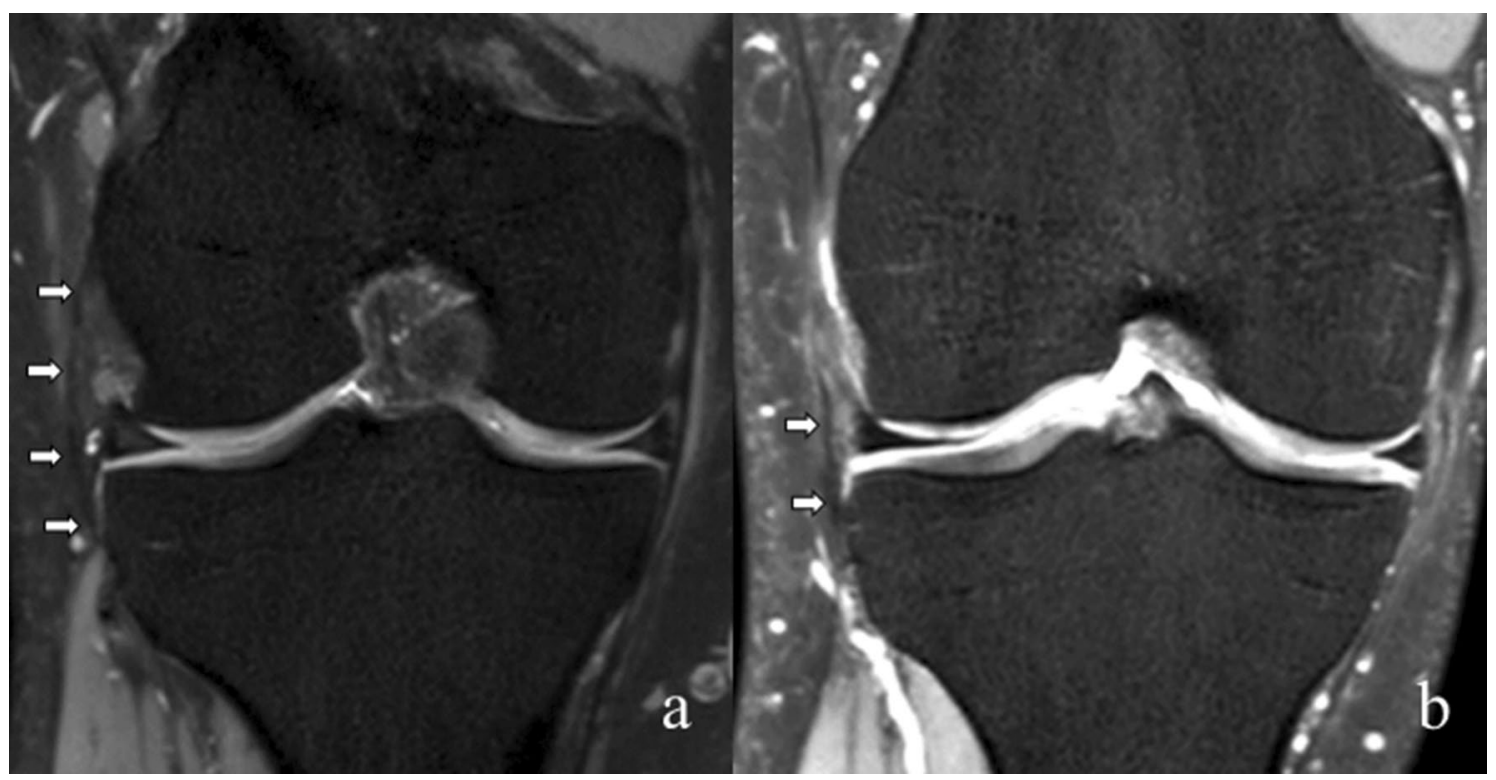

Figure 2. Standard coronal magnetic resonance images of right knees with the anterolateral ligament (ALL) (bold arrows) completely visible (a) with femoral and tibial insertion as well as the meniscal portion. The right image shows a partially visible (b) ALL.

significantly $(\mathrm{p}<0.001)$ with the application of MRP in both reads of both readers. The median distance of the tibial insertion of the ALL to the bony joint line, Gerdy's tubercle and the tip of the fibular head was $9 \mathrm{~mm}$ (range 6-13), $21 \mathrm{~mm}$ (range 11-30) and $25 \mathrm{~mm}$ (range 14-34), respectively.

The intraobserver reliability for the measurements of the distances of the tibial ALL insertion to the bony joint line, Gerdy's tubercle and the tip of the fibular head was good to excellent for reader 1 (ICC: 0.720; 0.799; 0.828 ) and fair to excellent for reader 2 (ICC: $0.544 ; 0.847 ; 0.885$ ). The interobserver reliability regarding the same distances was good to excellent (ICC: $0.612 ; 0.645 ; 0.757)$.

\section{Discussion}

This study aimed to establish an MRI evaluation protocol improving the visualization of the ALL using a standardized multiplanar reformation (MPR) protocol. It was hypothesized that visualization of the ALL can be improved significantly by applying this protocol when compared to the evaluation on standard coronal plane MRI. This analysis of 47 knee MRIs shows a significantly better visualization of the ALL after applying the MPR protocol described above, which confirms our hypothesis.

For many years the existence of a distinct ligament in the anterolateral knee capsule called the ALL has been questioned. Today, reliable anatomic, histologic and biomechanical data exist proofing the existence of the $\mathrm{ALL}^{17,22}$. More recently, the ALL related debate has shifted towards the clinical relevance of this structure ${ }^{2}$ including controversial discussions on the need for ALL reconstruction when anterior cruciate ligament reconstruction is performed ${ }^{23,24}$. Other possibilities to address the anterolateral rotatory instability are lateral tenodesis of parts of the Iliotibial band with modified Lemaire's or modified MacIntosh's techniques being the most referred ones $^{25,26}$. However, there is no clear data available to guide surgical planning yet.

Several authors have claimed to be able to visualize the ALL on standard MRI sequences ${ }^{10,11,27,28}$. After acute ACL injuries Ferretti et al. showed a high prevalence (88\%) of ALL abnormalities on MRI, which were significantly associated with lateral joint capsule tears. There were no problems reported in identifying the ALL independent of whether the ALL was injured or not ${ }^{28}$. This is in concordance with many other studies reporting that it is possible to characterize the ALL on MRI in acute case in a high percentage and could also link the MRI findings to the results of surgical exploration during ACL reconstruction ${ }^{28-30}$. Another study investigating the same topic stated that the ALL was not visible in $24 \%$ of cases $^{27}$. Helito et al. examined MRI scans of uninjured knees and reported a visibility of the whole ALL in $72 \%$ on standard MRI images ${ }^{12}$. A comparative table showing the visualization rates of recent MRI studies about the ALL can be found in the review of Andrade et al. ${ }^{14}$. Porrino et al. stated that in many MRI evaluations different anatomical structures are named as ALL and that a reliable discrimination of the anterolateral structures is very difficult if not impossible on routine $\mathrm{MRI}^{31}$. Another drawback of the studies mentioned above is their lack to specify the MRI layer thickness. MRI layer thickness may be a critical factor in identifying the ALL. Large slice thickness can obscure the delicate ALL due to partial volume artifacts/effects by adjacent ligamentous and capsule structures falling within the same voxels during MRI acquisition ${ }^{32}$. In the authors' opinion it should not be more than $1 \mathrm{~mm}$. This is in agreement with a study by Taneja et al. which reported a visibility rate of the ALL of no more than $11 \%$ when MRI with a layer thickness of $3 \mathrm{~mm}$ were performed ${ }^{13}$.

This overview of MRI studies on the ALL points out the problems associated with its visualization and explains the varying results. The ALL is a very thin structure that may be mistaken easily for the capsule or the meniscotibial and meniscofemoral attachments. Moreover, the ALL lies in very close proximity to the capsulo-osseus 


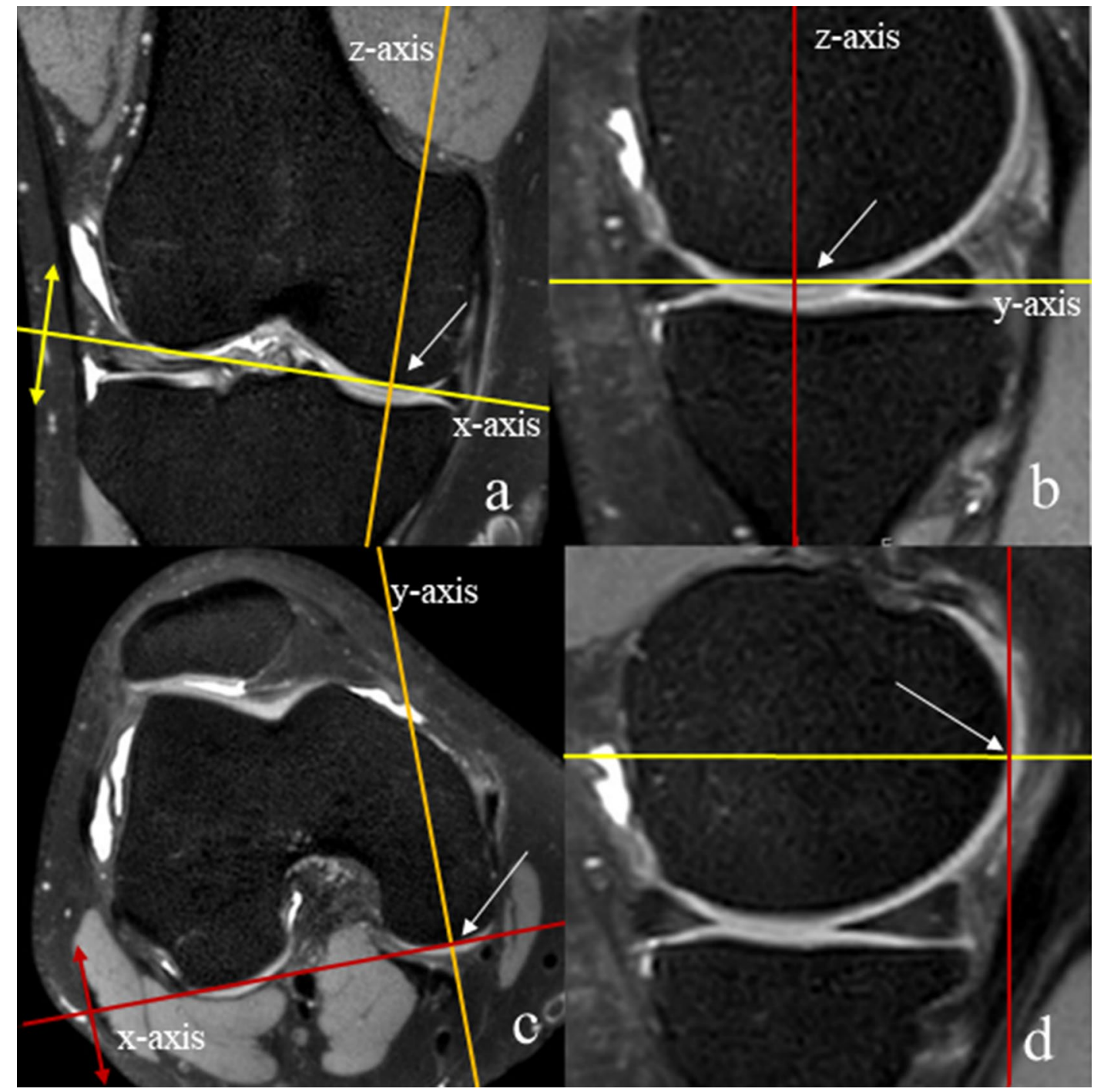

Figure 3. Alignment of the axial plane (a,b; yellow lines) and the coronal plane (c,d; red lines). The yellow line representing the axial plane was tangentially aligned to the femoral condyles inferiorly and posteriorly. This alignment was performed in the axial, coronal and sagittal plane. The white arrow marks the center of rotation of the 3D coordinate system.

layer of the ITB, which is connected to the femur at the intermuscular septum by Kaplan fibers ${ }^{33,34}$. Therefore, on MRI this may give the impression of a continuous structure inserting at the tibia and femur. However, the femoral insertion of these fibers is slightly proximal and posterior to the origin of the ALL, but confusion of these structures on MRI is possible. Therefore, in our opinion, a reliable identification and evaluation can only be ensured, if the whole ALL is visible as a distinct ligamentous structure with clear tibial and femoral attachment on a single MRI image and with a femoral attachment right next to the lateral epicondyle. To reach this goal we applied multiplanar reformation and a novel MRI evaluation protocol based on anatomic landmarks of the ALL. With this technique, the full visibility of the ALL could be improved from $36 \%$ on standard coronal MRI images to $94 \%$. However, it must be noted, that even using this technique the visualization of the ALL remains challenging and can only be accomplished by applying the knowledge of anatomical studies.

To be sure that the ALL was identified correctly, we rechecked our results by measuring the distances of its tibial insertion to the bony joint line, the middle of the Gerdy's tubercle and to the tip of the fibular head. These were found to be $9 \mathrm{~mm}, 21 \mathrm{~mm}$ and $25 \mathrm{~mm}$, respectively, which is within the range of the reported distances in anatomic studies ${ }^{17-19}$. A similar study using a 3D protocol was introduced by Muramatsu et al. in 2018, in this study a reference plane through the lateral epicondyle and a point midway between the posterior edge of Gerdy's tubercle and the anterior margin of the fibula was chosen. The authors claimed to be able to visualize the ALL in $100 \%$ of the uninjured cases using this technique ${ }^{16}$. Our study confirms this findings that the ALL can be visualized in a high percentage using a 3D technique. An advantage of the technique described in our work is, that it primarily uses the femoral condyles to align the MRI in a neutral rotation and neutral varus/valgus and therefore creates a reproducible starting point. Moreover, our reference plane did not go exactly through defined anatomical landmarks, but was oriented exactly to the course of the ALL using those landmarks only as 


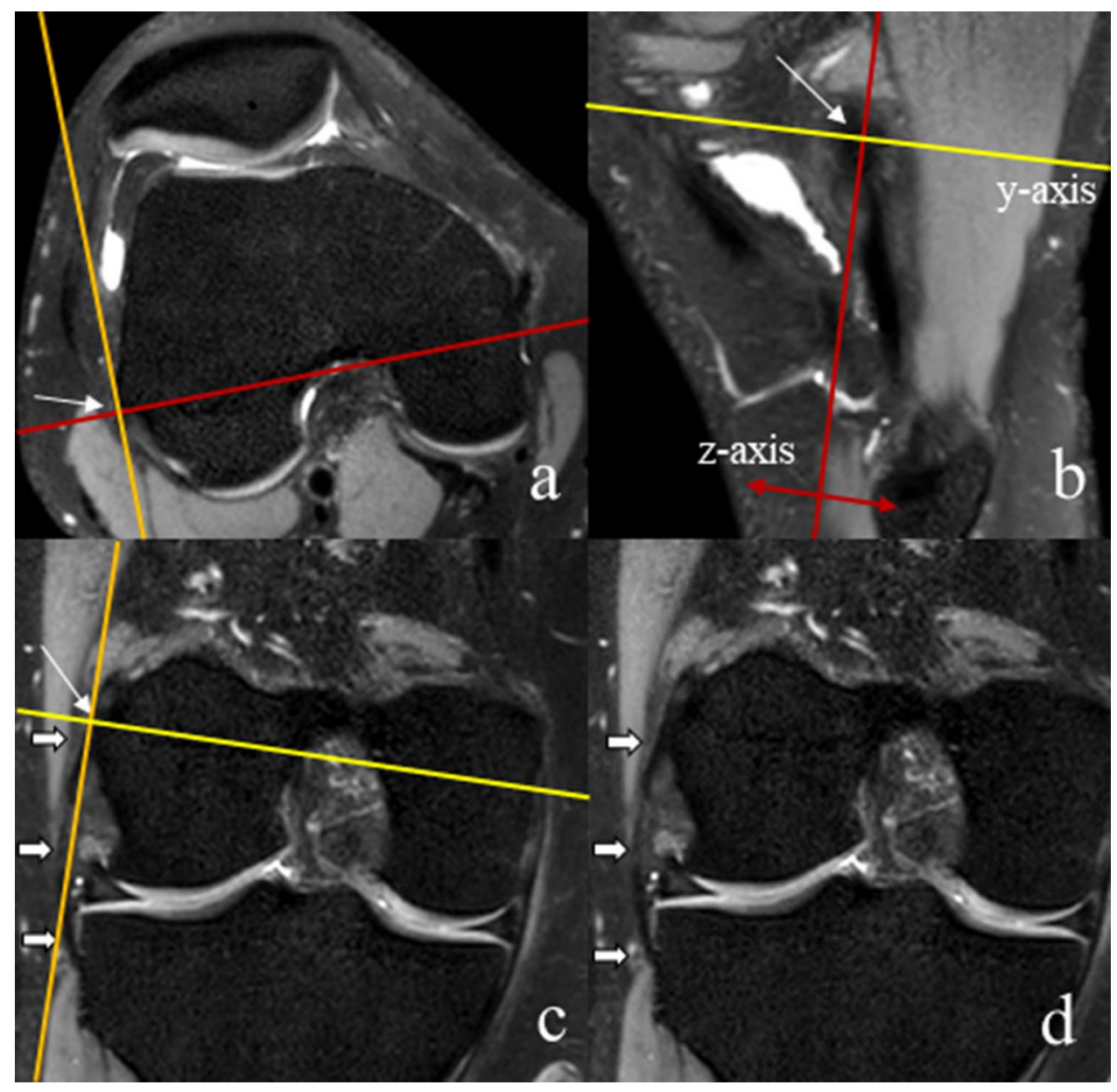

Figure 4. Visualization of the anterolateral ligament (ALL), using multiplanar reformation (MPR) with all planes visible and connected to each other, by tilting the coronal plane in the sagittal image (b; z-axis) with the center of rotation at the lateral epicondyle (origin of MPR coordinate system; $\mathbf{a}-\mathbf{c}$; white arrow). This results in full visibility of the ALL in the coronal image (c,d; white bold arrows). The red arrows indicate the possible directions of tilting the coronal plane around the center of rotation.

\begin{tabular}{|l|c|c|c|c|}
\hline & \multicolumn{2}{|l|}{ Reader 1 } & Reader 2 \\
\cline { 2 - 5 } & Read 1 & Read 2 & Read 1 & Read2 \\
\hline Standard coronal images \\
\hline Not visible & $4(9 \%)$ & $4(9 \%)$ & $2(4 \%)$ & $1(2 \%)$ \\
\hline Partially visible & $31(66 \%)$ & $30(64 \%)$ & $26(55 \%)$ & $23(49 \%)$ \\
\hline Completely visible & $12(26 \%)$ & $13(28 \%)$ & $19(40 \%)$ & $23(49 \%)$ \\
\hline MPR & $1(2 \%)$ & $1(2 \%)$ & $6(13 \%)$ & $3(6 \%)$ \\
\hline Partially visible & $46(98 \%)$ & $46(98 \%)$ & $41(87 \%)$ & $44(94 \%)$ \\
\hline Completely visible &
\end{tabular}

Table 1. Summary of the visibility of the ALL on standard coronal and MPR images.

a starting point. With this flexible reference plane the authors tried to address the variations of the femoral and tibial insertions of the ALL.

A major limitation of this study is, that the authors only had access to the MRI data and not to any clinical evaluation or additional demographic data like weight and height. Therefore absolute measurements of the dimensions of the ALL were not reasonable because they are directly linked for example to patient's height and need to be interpreted in that context. Another limitation is, that the described MPR technique cannot be utilized in standard $3 \mathrm{~mm}$ 2D MRI sequences, but needs 3D sequences with maximum $1 \mathrm{~mm}$ slice thickness. Moreover, we did not dived the visualization of the ALL in tibial, menical and femoral part as some authors proposed. The reason for this is that many authors claimed that the meniscal and femoral part of the ALL cannot be reliably 
identified on MRI. Therefor we decided to stay with "partially visible" which is less precise but can be stated more reliable. The goal of this study was to visualize the entire ALL on one MRI image, which could be achieved in $94 \%$ of the case using our MPR technique. The question about the parts of the ALL will become more important when the technique described here is used for the assessment of ALL injuries. We will address this in further studies.

\section{Conclusion}

Complete visualization of the ALL is critical for its identification and evaluation. Applying multiplanar reformation achieved reliable full-length visualization of the ALL in $94 \%$ of cases. The described MPR technique can be applied easily and fast in clinical routine. It is a reliable tool to improve the assessment of the ALL.

Received: 25 November 2020; Accepted: 14 June 2021

Published online: 24 June 2021

\section{References}

1. Pomajzl, R., Maerz, T., Shams, C., Guettler, J. \& Bicos, J. A review of the anterolateral ligament of the knee: current knowledge regarding its incidence, anatomy, biomechanics, and surgical dissection. Arthroscopy 31, 583-591 (2015).

2. Zaffagnini, S. et al. The anterolateral ligament does exist: An anatomic description. Clin. Sports Med. 37, 9-19 (2018).

3. Kraeutler, M. J., Welton, K. L., Chahla, J., LaPrade, R. F. \& McCarty, E. C. Current concepts of the anterolateral ligament of the knee: Anatomy, biomechanics, and reconstruction. Am. J. Sports Med. 46, 1235-1242 (2018).

4. Inderhaug, E., Stephen, J. M., Williams, A. \& Amis, A. A. Biomechanical comparison of anterolateral procedures combined with anterior cruciate ligament reconstruction. Am. J. Sports Med. 45, 347-354 (2017).

5. Rasmussen, M. T. et al. An in vitro robotic assessment of the anterolateral ligament, part 1: Secondary role of the anterolateral ligament in the setting of an anterior cruciate ligament injury. Am. J. Sports Med. 44, 585-592 (2016).

6. Sobrado, M. F. et al. Outcomes after isolated acute anterior cruciate ligament reconstruction are inferior in patients with an associated anterolateral ligament injury. Am. J. Sports Med. 48, 3177-3182 (2020).

7. Van der Watt, L. et al. The structure and function of the anterolateral ligament of the knee: A systematic review. Arthroscopy 31, 569-582 e563 (2015)

8. Getgood, A. et al. The anterolateral complex of the knee: Results from the International ALC Consensus Group Meeting. Knee Surg. Sports Traumatol. Arthrosc. 27, 166-176 (2019).

9. Shybut, T. B. et al. Effect of lateral meniscal root tear on the stability of the anterior cruciate ligament-deficient knee. Am. J. Sports Med. 43, 905-911 (2015).

10. Van Dyck, P. et al. Anterolateral ligament abnormalities in patients with acute anterior cruciate ligament rupture are associated with lateral meniscal and osseous injuries. Eur. Radiol. 26, 3383-3391 (2016).

11. Duran, S. et al. Evaluation of the anterolateral ligament of the knee by magnetic resonance imaging in patients with chronic anterior cruciate ligament rupture. J. Clin. Orthop. Trauma 10, 706-709 (2019).

12. Helito, C. P. et al. MRI evaluation of the anterolateral ligament of the knee: Assessment in routine 1.5-T scans. Skelet. Radiol. 43, 1421-1427 (2014).

13. Taneja, A. K. et al. MRI features of the anterolateral ligament of the knee. Skelet. Radiol. 44, 403-410 (2015).

14. Andrade, R. et al. Identification of normal and injured anterolateral ligaments of the knee: A systematic review of magnetic resonance imaging studies. Arthroscopy 35, 1594-1613 e1591 (2019).

15. Helito, C. P. et al. Magnetic resonance imaging assessment of the normal knee anterolateral ligament in children and adolescents. Skelet. Radiol. 47, 1263-1268 (2018).

16. Muramatsu, K. et al. Three-dimensional magnetic resonance imaging of the anterolateral ligament of the knee: An evaluation of intact and anterior cruciate ligament-deficient knees from the Scientific Anterior Cruciate Ligament Network International (SANTI) Study Group. Arthroscopy 34, 2207-2217 (2018).

17. Ariel de Lima, D. et al. Anatomy of the anterolateral ligament of the knee: A systematic review. Arthroscopy 35, 670-681 (2019).

18. Claes, S. et al. Anatomy of the anterolateral ligament of the knee. J. Anat. 223, 321-328 (2013).

19. Kennedy, M. I. et al. The anterolateral ligament: An anatomic, radiographic, and biomechanical analysis. Am. J. Sports Med. 43, 1606-1615 (2015).

20. Patel, R. M. \& Brophy, R. H. Anterolateral ligament of the knee: Anatomy, function, imaging, and treatment. Am. J. Sports Med. 46, 217-223 (2018).

21. Cicchetti, D. Guidelines, criteria, and rules of thumb for evaluating normed and standardized assessment instrument in psychology. Psychol. Assess. 6, 284-290 (1994).

22. Chahla, J., Geeslin, A. G., Cinque, M. E. \& LaPrade, R. F. Biomechanical proof for the existence of the anterolateral ligament. Clin. Sports Med. 37, 33-40 (2018).

23. Chahla, J., Menge, T. J., Mitchell, J. J., Dean, C. S. \& LaPrade, R. F. Anterolateral ligament reconstruction technique: An anatomicbased approach. Arthrosc. Tech. 5, e453-457 (2016).

24. Noyes, F. R., Huser, L. E., Jurgensmeier, D., Walsh, J. \& Levy, M. S. Is an anterolateral ligament reconstruction required in ACLreconstructed knees with associated injury to the anterolateral structures? A robotic analysis of rotational knee stability. Am. J. Sports Med. 45, 1018-1027 (2017).

25. Amirault, J. D., Cameron, J. C., MacIntosh, D. L. \& Marks, P. Chronic anterior cruciate ligament deficiency. Long-term results of MacIntosh's lateral substitution reconstruction. J. Bone Joint Surg. Br. 70, 622-624 (1988).

26. Jesani, S. \& Getgood, A. Modified Lemaire Lateral Extra-Articular Tenodesis Augmentation of Anterior Cruciate Ligament Reconstruction. JBJS Essent Surg Tech. 9 (2019).

27. Claes, S., Bartholomeeusen, S. \& Bellemans, J. High prevalence of anterolateral ligament abnormalities in magnetic resonance images of anterior cruciate ligament-injured knees. Acta Orthop. Belg. 80, 45-49 (2014).

28. Ferretti, A. et al. High prevalence of anterolateral ligament abnormalities on MRI in knees with acute anterior cruciate ligament injuries: A case-control series from the SANTI Study Group. Orthop. J. Sports Med. 7, 2325967119852916 (2019).

29. Monaco, E. et al. Correlation between magnetic resonance imaging and surgical exploration of the anterolateral structures of the acute anterior cruciate ligament-injured knee. Am. J. Sports Med. 47, 1186-1193 (2019).

30. Puzzitiello, R. N., Agarwalla, A., Zuke, W. A., Garcia, G. H. \& Forsythe, B. Imaging diagnosis of injury to the anterolateral ligament in patients with anterior cruciate ligaments: Association of anterolateral ligament injury with other types of knee pathology and grade of pivot-shift examination: A systematic review. Arthroscopy 34, 2728-2738 (2018).

31. Porrino, J. Jr. et al. The anterolateral ligament of the knee: MRI appearance, association with the Segond fracture, and historical perspective. AJR Am. J. Roentgenol. 204, 367-373 (2015).

32. Gonzalez Ballester, M. A., Zisserman, A. P. \& Brady, M. Estimation of the partial volume effect in MRI. Med. Image Anal. 6, 389-405 (2002). 
33. Batty, L. et al. The kaplan fibers of the iliotibial band can be identified on routine knee magnetic resonance imaging. Am. J. Sports Med. 47, 2895-2903 (2019).

34. Godin, J. A. et al. A comprehensive reanalysis of the distal iliotibial band: Quantitative anatomy, radiographic markers, and biomechanical properties. Am. J. Sports Med. 45, 2595-2603 (2017).

\section{Acknowledgements}

We thank ZIMMER BIOMET for supporting the first author's fellowship, during which this study was conducted.

\section{Author contributions}

A.H. and R.J.E. performed the measurements and drafted the manuscript. C.S.L. and E.F.L. analyzed the data and reviewed the manuscript. F.M.K. designed the study and helped to draft the manuscript. All authors reviewed the final manuscript and agree to be responsible for all aspects of the work.

\section{Competing interests}

The authors declare no competing interests.

\section{Additional information}

Correspondence and requests for materials should be addressed to A.H.

Reprints and permissions information is available at www.nature.com/reprints.

Publisher's note Springer Nature remains neutral with regard to jurisdictional claims in published maps and institutional affiliations.

(c) (i) Open Access This article is licensed under a Creative Commons Attribution 4.0 International License, which permits use, sharing, adaptation, distribution and reproduction in any medium or format, as long as you give appropriate credit to the original author(s) and the source, provide a link to the Creative Commons licence, and indicate if changes were made. The images or other third party material in this article are included in the article's Creative Commons licence, unless indicated otherwise in a credit line to the material. If material is not included in the article's Creative Commons licence and your intended use is not permitted by statutory regulation or exceeds the permitted use, you will need to obtain permission directly from the copyright holder. To view a copy of this licence, visit http://creativecommons.org/licenses/by/4.0/.

(C) The Author(s) 2021 